Indexed by

\title{
INCREASING THE EFFICIENCY OF MEMBRANE
Scopus'
TECHNOLOGIES IN WATER TREATMENT PROCESSES
}

\section{Kuvardina Elena Mikhailovna}

Southwest State University,

Crossref Faculty of Construction and Architecture, Department of Heat, Gas and Water Supply, Kursk, Russia

Key words: membrane technologies, vibration, water conditioning, reagents, ultrafiltration, selectiveness of membranes

\section{Cite article:}

Kuvardina, E. M. [2021]. Increasing the efficiency of membrane technologies in water treatment processes. Journal of Applied Engineering Science, 19(1) 172 - 174. DOI:10.5937/jaes0-30066 


\title{
INCREASING THE EFFICIENCY OF MEMBRANE TECHNOLOGIES IN WATER TREATMENT PROCESSES
}

\author{
Kuvardina Elena Mikhailovna* \\ Southwest State University, Faculty of Construction and Architecture, Department of Heat, Gas and Water \\ Supply, Kursk, Russia
}

The twenty-first century is the century of the rapid development of nanotechnologies, which determine the successful dynamics of the country's economy, save energy resources, and ensure a safe ecological level of the environment. Membrane processes have rightfully occupied their niche in various areas of a number of industries. Modern technologies have made it possible to create a wide range of membranes with good mechanical, thermal and chemical properties. The range of membrane devices and installations is also diverse, which are more and more improved from year to year. Water treatment of boiler houses is a necessary process that ensures successful commissioning of technological equipment of industrial enterprises, as well as heating systems. Water treatment systems are high-cost technologies, and these costs increase annually due to annually increasing trends: an increase in tariffs for water use, deterioration of water quality indicators in sources suitable for industrial use, stricter standards for quantitative and qualitative indicators for discharged effluents, increasing quality requirements treated water used in the technological cycle.

Key words: membrane technologies, vibration, water conditioning, reagents, ultrafiltration, selectiveness of membranes

\section{INTRODUCTION}

High-quality water treatment is the guarantor of trouble-free operation of boilers of the boiler house and other heating equipment throughout the entire production cycle and heating season [1 to 3].

Water is supplied to the boiler room either from autonomous sources (artificial water intakes), or from natural sources (rivers, ponds, etc.). The main negative property of these waters is the so-called "hardness". Moreover, the hardness of surface waters, as a rule, is less than the hardness of groundwater. The hardness of surface water is subject to significant seasonal fluctuations, usually reaching the highest value at the end of winter and the lowest during the flood period, when it is abundantly diluted with soft rain and melt water.

The concept of water hardness is usually associated with calcium cations $(\mathrm{Ca} 2+)$ and, to a lesser extent, magnesium $(\mathrm{Mg} 2+)$. In fact, all divalent cations affect the hardness to one degree or another. They interact with anions, forming compounds (hardness salts) capable of precipitating, forming a layer of scale on the inner surface of the boiler. The thickness of the scale layer of $1 \mathrm{~mm}$ entails an overconsumption of $5-8 \%$ of fuel, and poor-quality water treatment (or its absence) can lead to a decrease in efficiency systems by $15-30 \%$.

Contamination of heat exchange surfaces with scale deposits not only reduces the efficiency of the boiler house and requires periodic shutdowns to clean boilers and other heat exchange equipment, but also causes a chain of economic losses in the production, transportation and consumption of heat.

Modern boiler houses successfully use membrane tech- nologies at various stages of water purification, from filtration membranes to reverse osmosis membranes.

\section{EXPERIMENTAL PART AND PROCESSING OF RESULTS}

When operating a membrane of any type, one problem always arises - the formation of a layer of deposits on the membrane surface, which slows down or completely stops its operation, Fig. 1. [3 to 5] This is especially true for solutions with a high content of small mechanical impurities or high-molecular compounds.

The resulting layer of scurf (sediments) occurs as a result of the phenomenon of concentration polarization. Preliminary purification of solutions carried out before membrane separation leads to an increase in the hardware design of the technological cycle, and, consequently, to an increase in the cost of the output product.

There are a number of ways to prevent or reduce the effect of the concentration polarization process, associated mainly with the design features of the apparatus. But a change in the design of membrane devices cannot fully

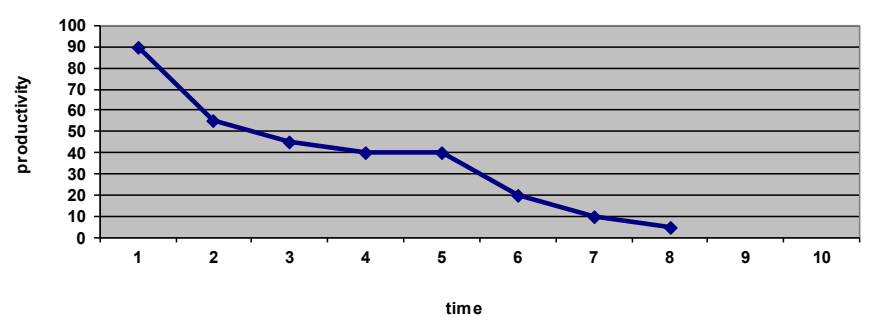

Figure 1: Dependence of the productivity of the ultrafiltration process on time.

(results are presented in dimensionless form) 
solve the problem that has arisen. To eliminate it, technological methods are required that affect the operation of membranes in order to intensify them.

Membrane separation processes have the peculiarity of changing in time the rate of passage of the liquid phase (solvent) through the membrane partition. As indicated in $[3,6,7]$, the dynamics of the ultrafiltration process is conventionally divided into three periods. The initial period is characterized by a decrease in the filtration flow, due to the appearance of osmotic pressure caused by the concentration of the dispersed phase at the membrane surface, as well as blockage of the largest pores of the membrane. The drop in permeability can proceed by different mechanisms, but with the obligatory transition of the ultrafiltration process to the second period - the period of formation of a layer of sediment on the membrane surface, called the period of stationary operation of the membrane. The third period is associated with the formation of a stable layer of deposits on the surface of the membrane and sorption in the pores with the formation of gel layers of dispersed inclusions in them - the decay period of the process.

An increase in the tangential flow velocity decreases the thickness of the laminar boundary layer, increases the intensity of mixing in the near-wall region of the membrane, and decreases the rate of formation of the sediment layer. At the same time, periodic processes occurring between the core of the stream and the near-wall boundary layer, under certain conditions, lead to the formation of standing waves, which create a destructive effect on the sediment layer. However, the resulting standing waves are not stable and this method only reduces the rate of formation of a layer of unwanted deposits on the membrane surface.

Let us dwell in more detail on the method of water purification from high-molecular compounds and fine mechanical particles, when the solution is passed through the intermembrane gap under an average overpressure of up to $0.5 \mathrm{MPa}$, an average speed of up to $5 \mathrm{~m} / \mathrm{s}$, and a vibrating device is introduced into the flow of pressure pulsations and the flow of resonance frequency.

Thus, the formation of a standing wave in the intermembrane channel is forced by means of a vibrating device connected to the flow at the entrance to the membrane apparatus. Pulsations introduced into the flow by a vibrating device create additional flow turbulization.

The vibrations created by the electrodynamic vibration exciter are transmitted directly into the flow to be separated and create a speed $\mathrm{V}$, which changes according to the harmonic law:

$V=V_{0}+\Delta V \sin \omega t$

where is $\Delta \mathrm{V}$ - amplitude of velocity fluctuations.

This leads to intense fluctuations in the flow rate and pressure along the entire path of the solution through the entire apparatus. In the intermembrane channels, favorable hydrodynamic conditions are formed for the destruc-

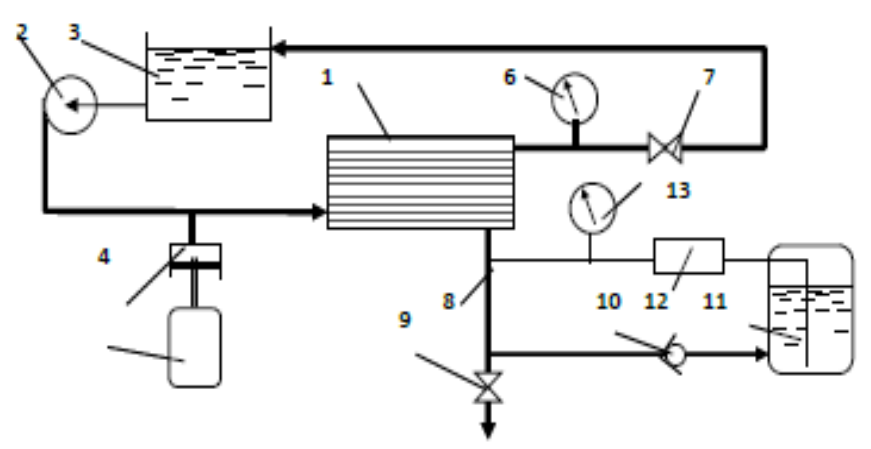

Figure 2: Diagram of an experimental setup with an electrodynamic vibration exciter.

1-membrane apparatus; 2-pump; 3-intermediate tank; 4-vibration transmission unit; 5- vibration

generator; 6.13-gauge; 7.9 valve; 8-line of filtrate

removal; 10-check valve; 11-hydropneumatic accumulator; 12-vibration interrupter

tion of unwanted deposits that form on the membrane surface. The installation diagram is shown in Figure 2.

The solution to be separated enters the intermembrane channel through the CA permeable boundary at a speed $\mathrm{V}$, passes through the channel, in which it is filtered on the CK semipermeable membrane and exits from the BK permeable boundary, with a high content of the solid phase of mechanical impurities in its volume. The filtrate passes through the semi-permeable boundary (membrane) of the CK. The solution flow diagram is shown in Figure 3.

In the course of numerical and experimental studies, it was found that when using vibrations in the ultrafiltration process, the dynamic curve of the process has only two pronounced periods, the initial and constant filtration rate, longer than similar sections of the dynamic curves for other types of feeding the separated solution (Fig. 4) [3 to 4]. This slows down or even prevents the formation of a layer of deposits on the membrane surface, which in turn leads to an increase in the productivity of the apparatus by almost $30 \%[3,8,9]$.

The influence of vibrations on the mechanical properties of the membrane was studied in the frequency range from 1500 to $10 \mathrm{~Hz}[3,10]$.

The highest performance in the specified limit corresponded to $67 \mathrm{~Hz}$. (Figure 5).

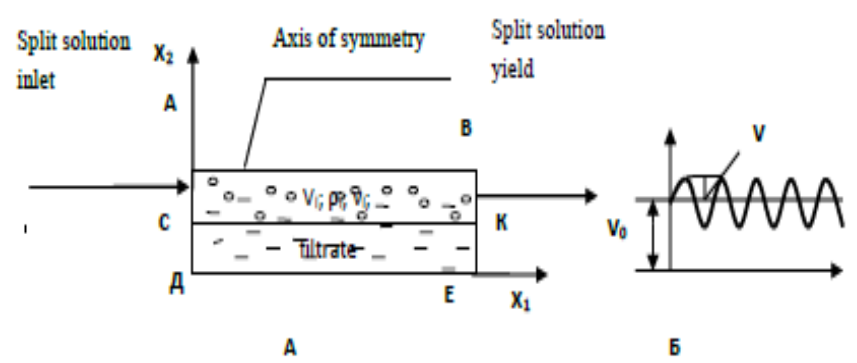

Figure 3: A- Scheme of the passage of juice through the intermembrane channel; $B$ - distribution of the movement of the solution to be separated under the influence of vibration introduced into the flow 


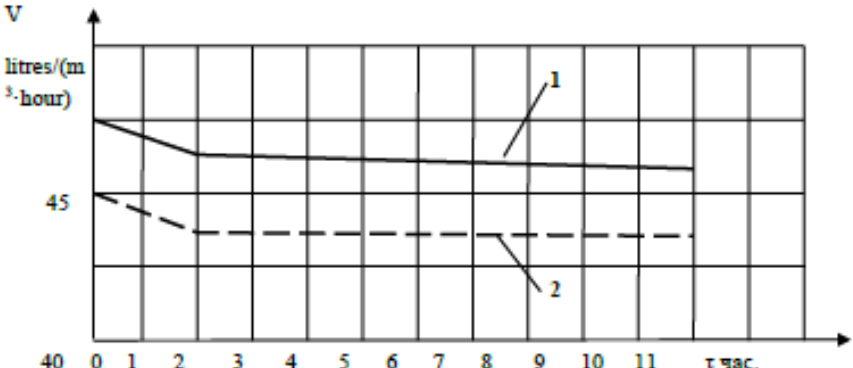

Figure 4: Kinetic curves of ultrafiltration in the presence of vibration

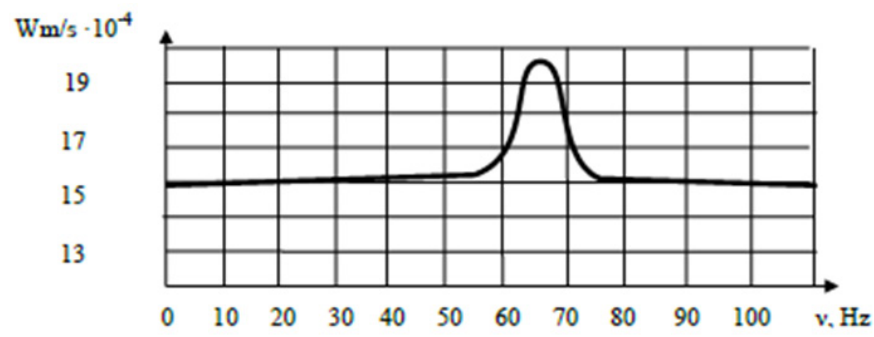

Figure 5: Dependence of the filtration speed on the frequency of oscillations introduced into the solution

The performance of the membrane at all values of the specified frequency range did not change, remaining within the performance of the membrane apparatus operating under pressure without introducing vibrations into the solution, and significantly increased when exposed to low frequencies in the range of $60-70 \mathrm{~Hz}$.

\section{CONCLUSION}

Based on the foregoing, we came to the conclusion that in this case a resonance phenomenon occurs when the natural frequency of the membrane elements coincides with the frequency of the pulsations of the electrodynamic vibration exciter introduced into the solution to be separated. Thus, in the section of constant filtration speed, stable standing waves are generated, generated by the vibrating device. This, in turn, prevents the formation of a layer of unwanted deposits on the membrane surface [3, 11, 12].

\section{REFERENCES}

1. Bezrukov, N., Bukhovets, E., et al. (2009). Integrated membrane technologies in water treatment: experience and implementation prospects. The III scientific-practical conference "Modern technologies of water treatment and protection of equipment from corrosion and scale formation." Moscow. Expocentre, 175 p.

2. Feng LIN,Shoubin ZHANG,Guoqiang MA,Liping QIU, Huajun SUN. (2018).Application of Ceramic Membrane in Water and Wastewater Treatment. E3S Web of Conferences. 3International Conference on Advances in Energy and Environment Research (ICAEER 2018), vol. 53, 4, DOI: 10.1051/e3sconf/20185304032.
3. Kuvardina, E. Prospects for membrane technologies for purification of multicomponent solutions: monograph. Kursk, $123 \mathrm{p}$

4. Yatsun, S., Kudryavtsev, V., Kuvardina, E. (2002). On the use of a pulsed feed of a separated solution during ultrafiltration of diffusion sugar beet juice. AllUkrainian scientific and technical journal. Vibrations in engineering and technology, no. 4 (25), 70-71.

5. Polivanova, T., Semicheva, N., Ryabtseva, S. (2020). Development of technology for reducing the technogenic impact of sugar factories on the environment of localities. Journal of Applied Engineering Science, vol. 18, br. 2, str. 238-242, DOI: 10.5937/jaes18-26301.

6. Dlamini, D.S., Tesha, J.M.,Vilakati, G.D., Mamba, B.B., Mishra, A.K., Thwala, J.M., Li, J.X. (2020). A critical review of selected membrane- and powder-based adsorbents for water treatment: Sustainability and effectiveness. Journal of Cleaner Production, vol. 2, 123497, DOI:10.1016/j.jclepro.2020.123497.

7. Yanar, N., Kallem, P., Son, M., Park, H., Kang, S., Choi, H. (2020).A New era of water treatment technologies: 3D printing for membranes. Journal of Industrial and Engineering Chemistry, vol. 91, 1-14, DOI:10.1016/j.jiec.2020.07.043.

8. Zhu, Z.Z., Chen, Z., Luo, X., Zhang, W.X., Meng, S.J.,(2020). Gravity-driven biomimetic membrane (GDBM): An ecological water treatment technology for water purification in the open natural water system. Chemical Engineering Journal, vol. 399, 125650, DOI:10.1016/j.cej.2020.125650.

9. Cooray, T., Wei, Y.S., Zhang, J.Y., Zheng, L.B., Zhong, H.,Weragoda, S.K.,Weerasooriya, R., (2019). Drinking-Water Supply for CKDu Affected Areas of Sri Lanka, Using Nanofiltration Membrane Technology: From Laboratory to Practice. Water, vol. 11, no. 12, 2512, DOI:10.3390/w11122512.

10. Zhu, X.B.,Jassby, D.,(2019). Electroactive Membranes for Water Treatment: Enhanced Treatment Functionalities, Energy Considerations, and Future Challenges. Accounts of Chemical Research, vol. 52, no. 5, 1177-1186, DOI: 10.1021/acs.accounts.8b00558.

11. Bredikhin V.V., Akulshin A.A., Bredikhina N.V., Pereverzeva V.S. (2017). A Method of Calculating Filtration Rate of an Infiltration Water Intake in Kursk Region. Journal of Applied Engineering Science, vol. 15, br. 3, str. 208-211, DOI:10.5937/jaes15-14644.

12. Akulshin A.A., Bredikhina N.V., Akulshin An.A., Aksenteva I.Y., Ermakova N.P.Development of Filters with Minimal Hydraulic Resistance for Underground Water Intakes. Civil Engineering Journal, vol. 6, no. 5, 919-927.

Paper submitted: 28.12.2020.

Paper accepted: 25.02.2021.

This is an open access article distributed under the CC BY 4.0 terms and conditions. 